\title{
Learning Illumination from Diverse Portraits
}

\author{
Chloe LeGendre \\ Google Research \\ chlobot@google.com
}

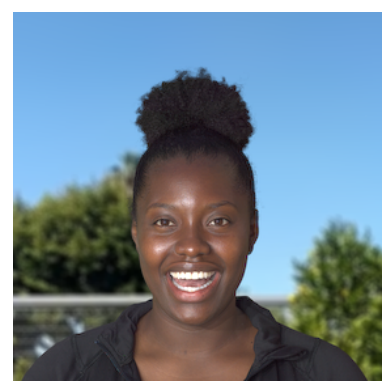

(a) Input portrait

\author{
Wan-Chun Ma \\ Rohit Pandey \\ Sean Fanello \\ Google
}

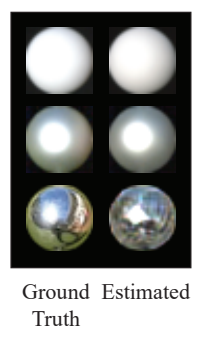

(b) Lighting

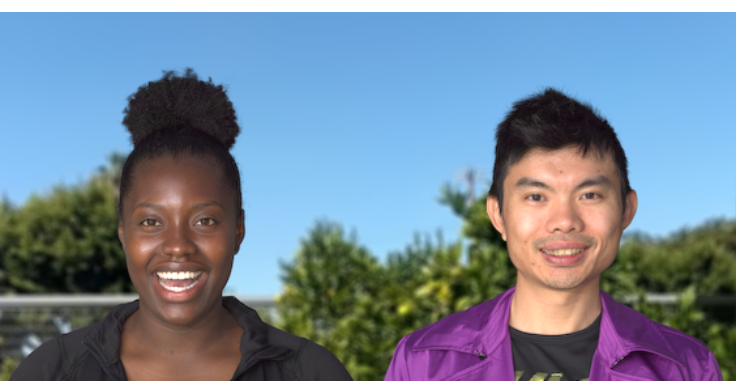

(c) Original and novel subjects lit with estimated lighting

\author{
Paul Debevec \\ Google Research
}

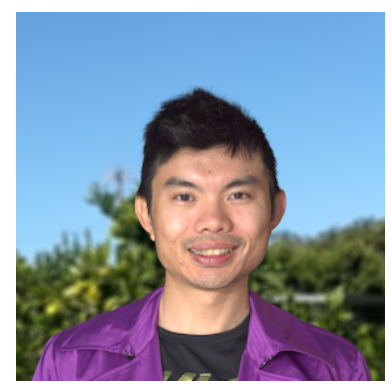

(d) Novel subject lit by GT lighting

Figure 1: Our network estimates $360^{\circ}$ HDR lighting from an LDR portrait. (a) Input portrait image. (b) Ground truth and estimated lighting, shown on diffuse, glossy, and mirror spheres. (c) Original and novel subjects lit consistently by the estimated lighting (using image-based relighting). (d) The novel subject lit with the original subject's ground truth lighting. For both subjects, the appearance under the estimated lighting closely matches that under the ground truth.

\begin{abstract}
We present a learning-based technique for estimating high dynamic range (HDR), omnidirectional illumination from a single low dynamic range (LDR) portrait image captured under arbitrary indoor or outdoor lighting conditions. We train our model using portrait photos paired with their ground truth illumination. We generate a rich set of such photos by using a light stage to record the reflectance field and alpha matte of 70 diverse subjects in various expressions. We then relight the subjects using image-based relighting with a database of one million HDR lighting environments, compositing them onto paired high-resolution background imagery recorded during the lighting acquisition. We train the lighting estimation model using rendering-based loss functions and add a multi-scale adversarial loss to estimate plausible high frequency lighting detail. We show that our technique outperforms the state-of-the-art technique for portrait-based lighting estimation, and we also show that our method reliably handles the inherent ambiguity between overall lighting strength and surface albedo, recovering a similar scale of illumination for subjects with diverse skin tones. Our method allows virtual objects and digital characters to be added to a portrait photograph with consistent illumination. As our inference runs in real-time on a smartphone, we enable realistic rendering and compositing of virtual objects into live video for augmented reality.
\end{abstract}

Permission to make digital or hard copies of all or part of this work for personal or classroom use is granted without fee provided that copies are not made or distributed for profit or commercial advantage and that copies bear this notice and the full citation on the first page. Copyrights for components of this work owned by others than ACM must be honored. Abstracting with credit is permitted. To copy otherwise, or republish, to post on servers or to redistribute to lists, requires prior specific permission and/or a fee. Request permissions from permissions@acm.org.

SA '20 Technical Communications, December 4-13, 2020, Virtual Event, Republic of Korea

(C) 2020 Association for Computing Machinery.

ACM ISBN 978-1-4503-8080-5/20/12 . \$ \$15.00

https://doi.org/10.1145/3410700.3425432

\section{CCS CONCEPTS}

- Computing methodologies $\rightarrow$ Image-based rendering; Image processing; Scene understanding.

\section{KEYWORDS}

lighting estimation, inverse lighting

\section{ACM Reference Format:}

Chloe LeGendre, Wan-Chun Ma, Rohit Pandey, Sean Fanello, Christoph Rhemann, Jason Dourgarian, Jay Busch, and Paul Debevec. 2020. Learning Illumination from Diverse Portraits. In SIGGRAPH Asia 2020 Technical Communications (SA '20 Technical Communications), December 4-13, 2020, Virtual Event, Republic of Korea. ACM, New York, NY, USA, 4 pages. https://doi.org/10.1145/3410700.3425432

\section{INTRODUCTION AND RELATED WORK}

The technique of blending real-world camera footage with computergenerated content is used extensively in both visual effects based films and mobile augmented reality (AR). In both scenarios, the realism of the composite depends on the consistency between the real-world lighting and that used to render the virtual content. Thus, computer graphics practitioners work painstakingly to capture and reproduce real-world illumination inside virtual sets. Debevec [1998] introduced a technique for real-world lighting capture, recording the color and intensity of omnidirectional illumination by photographing a mirror sphere using multiple exposures. However, in both film and AR scenarios, such lighting measurements are not always available, yet lighting artists must still reason about illumination using cues in the scene. If the footage includes faces, this task is somewhat less challenging, as faces include a diversity of surface normals and reflect light somewhat predictably.

Prior work has leveraged the strong geometry and reflectance priors from faces to solve for lighting from portraits. Since Marschner and Greenberg [1997] introduced portrait "inverse lighting," most 
such techniques [Egger et al. 2018; Knorr and Kurz 2014; Sengupta et al. 2018; Shim 2012; Tewari et al. 2017; Zhou et al. 2018] have sought to recover both facial geometry and a low frequency approximation of distant scene lighting, usually represented using up to a $2^{\text {nd }}$ order spherical harmonic (SH) basis. This approximation is justified by the fact that skin reflectance is predominantly diffuse (Lambertian) and thus acts as a low-pass filter on the incident illumination. For diffuse materials, irradiance indeed lies very close to a 9D subspace well-represented by this basis [Ramamoorthi and Hanrahan 2001]. However, to the skilled portrait observer, the capture-time lighting is revealed not only through the skin's diffuse reflection, but also through the directions and extent of cast shadows and the intensity and locations of specular highlights. Inspired by these cues, we train a neural network to perform inverse lighting from portraits, estimating omnidirectional HDR illumination without assuming any skin reflectance model. Our technique yields higher frequency lighting that can be used to convincingly render novel subjects into real-world portraits, with applications in both visual effects and AR when off-line lighting measurements are unavailable. Furthermore, our lighting inference runs in real-time on a smartphone, enabling such applications.

A few recent works have also sought to recover illumination from portraits using deep learning, including Sun et al. [2019] for arbitrary scenes and Calian et al. [2018] for outdoor scenes. We show that our method out-performs both and generalizes to arbitrary scenes. Yi et al. [2018] estimated specular highlights and ray-traced them into a panorama of lighting directions. However, this produced mostly empty panoramas, with only dominant light sources represented. In contrast, our estimated lighting is omnidirectional. As lighting estimation from unconstrained (non-portrait) images has also received considerable attention (e.g. [Gardner et al. 2017; Hold-Geoffroy et al. 2017; LeGendre et al. 2019]), we further compare to and outperform one such technique.

Lighting estimation is complicated by the inherent ambiguity between surface reflectance (albedo) and light source strength. Statistical priors for facial albedo have been leveraged to resolve this ambiguity [Calian et al. 2018; Egger et al. 2018], but, to the best of our knowledge, we are the first to explicitly evaluate the performance of our model on subjects with different skin tones. In contrast, Sun et al. [2019] report lighting accuracy with a scaleinvariant metric, while Calian et al. [2018] show results for rendered and photographed faces where the subjects are predominantly light in skin tone. We show that for a given lighting condition, our model can recover lighting at a similar scale across diverse subjects.

In summary, our contributions are the following:

- A deep learning method to estimate HDR illumination from LDR images of faces in both indoor and outdoor scenes. Our technique outperforms the previous state-of-the-art.

- A first-of-its-kind analysis that shows that our HDR lighting estimation technique reliably handles the ambiguity between light source strength and surface albedo, recovering similar illumination for subjects with diverse skin tones.

\section{METHOD}

We train our lighting estimation model in a supervised manner using a dataset of synthetic portraits and their corresponding ground truth illumination. To generate this dataset, we first photographed 70 diverse subjects in a light stage system as illuminated by 331 directional light sources forming a basis on a sphere (see Fig. 2), such that the captured subject can be relit to appear as they would in any scene with image-based relighting [Debevec et al. 2000].
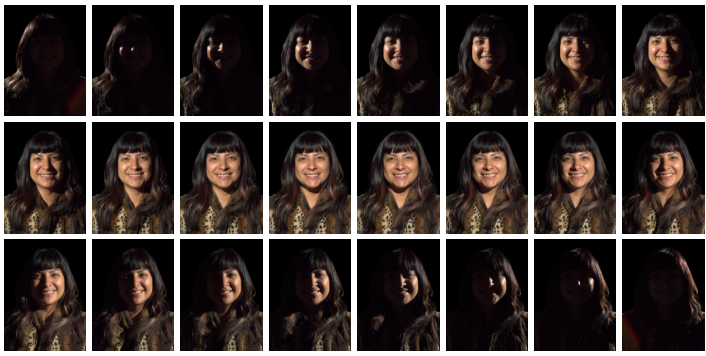

Figure 2: Example "One-Light-at-a-Time" images.

Each subject performed nine different facial expressions while wearing different accessories, yielding about 630 different sequences from six different camera views, totalling 3780 unique sequences. In addition to age and gender diversity, we were careful to photograph subjects spanning a wide range of skin tones (see Fig. 3). For two frontal camera views, we also acquired images to compute an alpha matte for each subject, using the technique of Debevec et al. [2002].

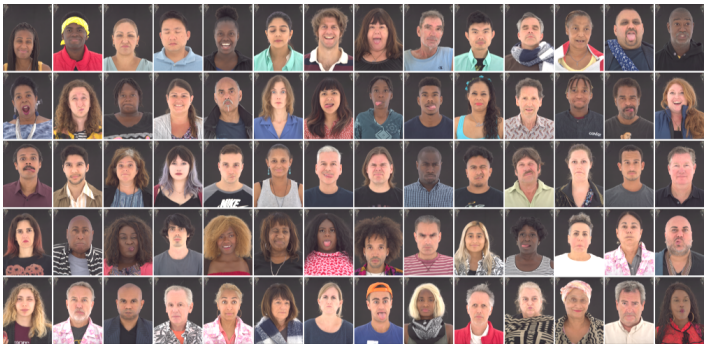

Figure 3: Example portraits of the 70 recorded subjects.

To relight the subjects, we require many HDR panoramas with no clipped light sources. We extend the LDR data collection technique of LeGendre et al. [2019] to capture 1 million indoor and outdoor lighting environments, promoting them to HDR via a novel nonnegative least squares solver before using them for relighting. For further details, please see our supplemental material. As this lighting capture technique also yields a high-resolution background image corresponding to the illumination, and since arbitrary images on their own contain useful lighting cues [Gardner et al. 2017; Hold-Geoffroy et al. 2017], we composite our relit subjects onto the these backgrounds rather than onto black as in Sun et al. [2019], as shown in Fig. 4, producing images which mostly appear to be natural photographs taken out in the wild. For each lighting environment, we randomly select 8 captures to relight from the training set (across subjects, facial expressions, and camera views), generating 8 million training portraits with illumination labels.

We crop the input images to a detected face region to avoid wasting network capacity learning a face detector and then clip and sRGB-encode the images. The crops are resized to $256 \times 256$ and normalized to the range of $[-0.5,0.5]$. We use an encoder-decoder architecture with a latent vector of size 1024 at the bottleneck, representing log-space HDR illumination. For further details, please see 


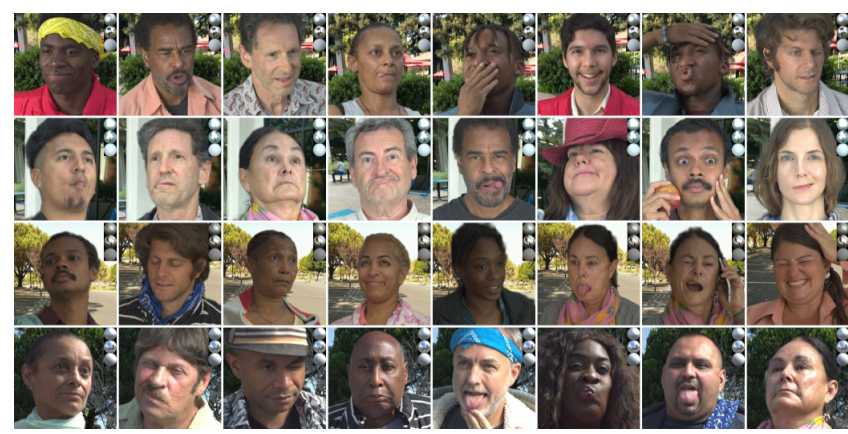

Figure 4: Example synthetic training portraits. Upper right corners: ground truth HDR illumination.

our supplemental material. For training, we employ the renderingbased and adversarial loss functions of LeGendre et al. [2019], extended to the multi-scale domain using MSG-GAN [Karnewar and Wang 2020]. The output is a $32 \times 32$ HDR image of a mirror ball representing log-space omnidirectional illumination.

\section{EVALUATION AND RESULTS}

Of the 70 subjects, we use 63 for training and 7 for evaluation, ensuring that all expressions and camera views for a given subject belong to the same subset. We manually select the 7 subjects to include both skin tone and gender diversity. We also capture evaluation lighting environments in new indoor and outdoor locations unseen in training, pairing these only with the evaluation subjects.

Comparisons. Accurately estimated lighting should correctly render objects with arbitrary reflectance properties, so we evaluate our model using an LDR $L_{1}$ reconstruction loss $\left(L_{\text {rec }}\right)$ on the renderings of three spheres (diffuse, matte silver, and mirror) using ground truth versus estimated illumination. In Table 1, we compare against Sun et al. [2019], Calian et al. [2018], and a $2^{\text {nd }}$ order SH decomposition of the ground truth lighting. We re-implement Sun et al. [2019], training the model on our data for a fair comparison. To compare with Calian et al. [2018], the authors generously computed outdoor lighting for a set of portraits. However, the scale of their lighting depends on an albedo prior, so, for a best case comparison, we re-scale the author-provided illumination to match the ground truth total scene radiance. Finally, we compare against the $2^{\text {nd }}$ order $\mathrm{SH}$ decomposition, as this represents the best case scenario for any monocular face reconstruction technique that uses this low frequency basis. Our model out-performs Sun et al. [2019] and Calian et al. [2018] for the diffuse and matte silver spheres. However, Sun et al. [2019] out-performs ours for the mirror sphere, as its logspace loss on lighting is similar to $L_{\text {rec }}$ for the mirror ball (but in HDR). The $2^{\text {nd }}$ order SH approximation of the ground truth illumination out-performs our model for $L_{\text {rec }}$ for the diffuse ball, as a low frequency representation of illumination suffices for rendering Lambertian materials. However, our model out-performs the $2^{\text {nd }}$ order SH decomposition for $L_{\text {rec }}$ for the other BRDFs, suggesting that our model is better suited for rendering diverse materials. In Fig. 6 we show qualitative results, rendering the three spheres using illumination produced by each method. The $2^{\text {nd }}$ order is again shown to be ill-suited to rendering non-Lambertian materials. For Calian et al. [2018], the sun direction is misrepresented, as our lighting environments include diverse camera elevations, with the horizon line not exclusively along the equator. Additional comparisons and an ablation study are provided in our supplemental material.

Table 1: Comparison among methods: Average $L_{1}$ loss for diffuse (d), mirror (m), and matte silver (s) spheres, for unseen indoor (UI) and outdoor (UO) locations. ( ${ }^{*} n=237$ for Calian et al. [2018] due to face tracking failures.)

\begin{tabular}{|c|c|c|c|c|c|c|}
\hline \multirow[b]{2}{*}{$n=270^{*}$} & \multicolumn{2}{|c|}{$L_{1(d)}$} & \multicolumn{2}{|c|}{$L_{1(s)}$} & \multicolumn{2}{|c|}{$L_{1(m)}$} \\
\hline & UI & UO & UI & UO & UI & UO \\
\hline Our model & 0.069 & 0.056 & 0.087 & 0.072 & 0.181 & 0.157 \\
\hline $2^{\text {nd }}$ order $\mathrm{SH}$ of GT & 0.016 & 0.015 & 0.120 & 0.109 & 0.306 & 0.247 \\
\hline Sun et al. [2019] & 0.145 & 0.120 & 0.113 & 0.100 & 0.154 & 0.139 \\
\hline Calian et al. [2018] & - & 0.158 & - & 0.163 & - & 0.215 \\
\hline
\end{tabular}

Lighting Consistency for Diverse Skin Tones. In Fig. 5, we report $L_{\text {rec }}$ for each of the three spheres individually, for unseen indoor and outdoor lighting environments for each evaluation subject. Each subject's dataset includes diverse camera viewpoints, facial expressions, and hats/accessories. While there are some slight variations in $L_{\text {rec }}$ across subjects, the model's performance appears similar across diverse skin tones. Although $L_{\text {rec }}$ is a useful metric, its absolute value operation masks the sign of residual error. To see whether radiance is missing or added to the predicted lighting for each subject, we also explore the total relative radiance difference and present further qualitative results in our supplemental material.

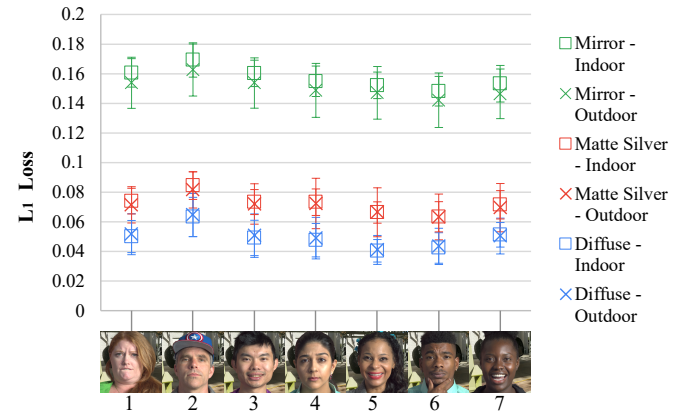

Figure 5: Average $L_{\text {rec }}$ for individual evaluation subjects, $n=$ 496 each, similar for subjects of diverse skin tones.

In Fig. 1, we show that for a given input portrait (Fig. 1a), and lighting estimated from this portrait using our method (Fig. 1b), we can accurately light a subject of a different skin tone (Fig. 1c) without adjusting the scale of the illumination and composite them into the original image, closely matching that subject's ground truth appearance (Fig. 1d). Additional such examples and results on in-the-wild portraits are included in our supplemental material.

Application: Mobile Augmented Reality. Our lighting inference runs in real-time on a mobile device (CPU: $27.5 \mathrm{fps}$, GPU: $94.3 \mathrm{fps}$ on a Google Pixel 4 smartphone), enabling real-time rendering and compositing of virtual objects for smartphone AR applications. We show our inference running in real-time in our supplemental video.

Application: Digital Double Actor Replacement. In Fig. 7, we estimate lighting from an in-the-wild portrait (a), and light a virtual character to composite into the original scene with consistent illumination (c). Our method can be used for digital double actor replacement, without on-set lighting measurements. 

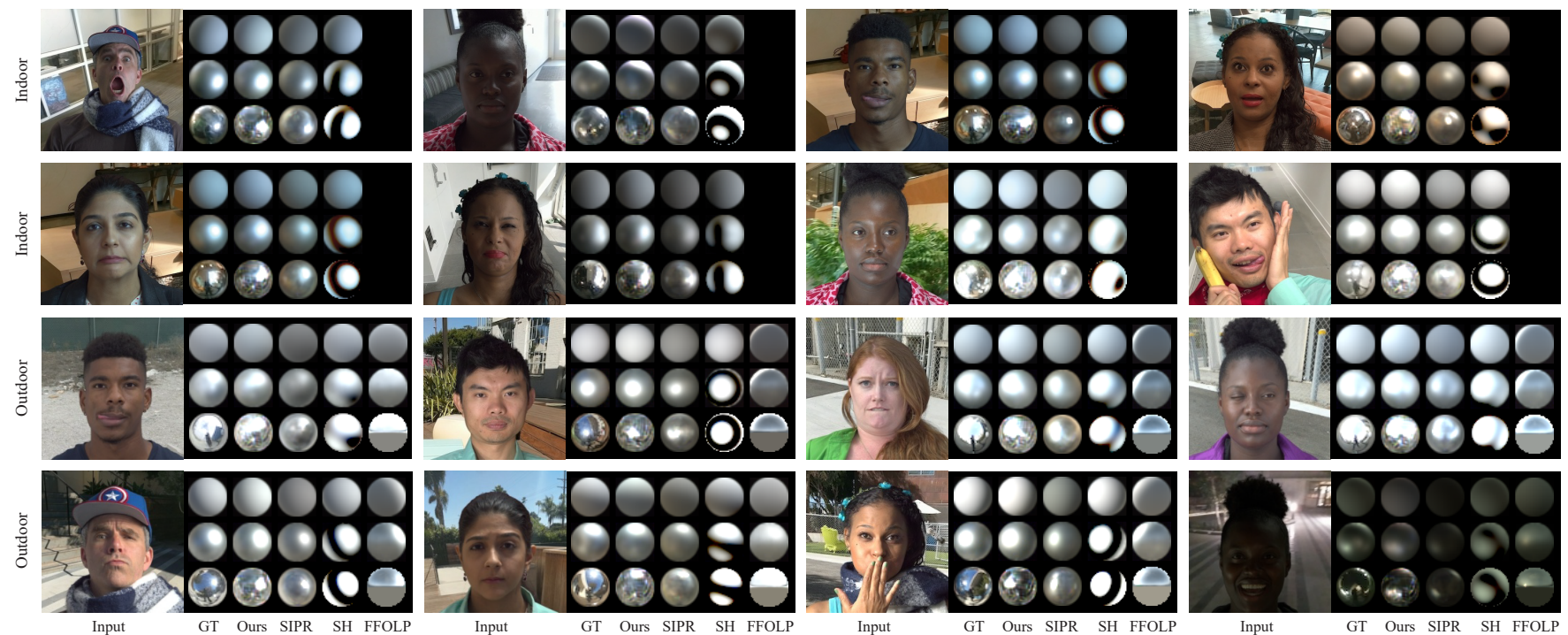

Figure 6: Sphere renderings (diffuse, matte silver, and mirror) for evaluation subjects in indoor and outdoor environments. We compare against SIPR [Sun et al. 2019], a $2^{\text {nd }}$ order SH decomposition of the ground truth illumination, and for outdoor scenes, radiance-scaled FFOLP [Calian et al. 2018]. Our model more faithfully recovers the total scene radiance compared with SIPR, and, unlike the SH decomposition, can be used to render non-Lambertian materials.

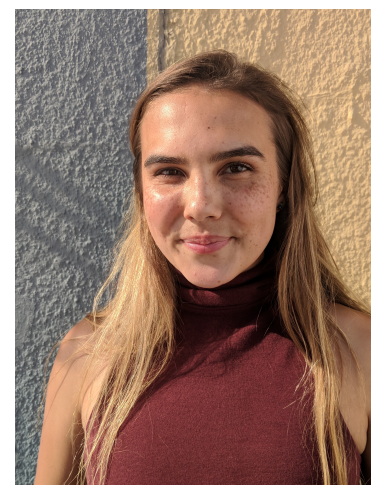

(a) input in-the-wild portrait

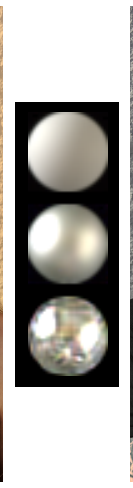

(b) lighting

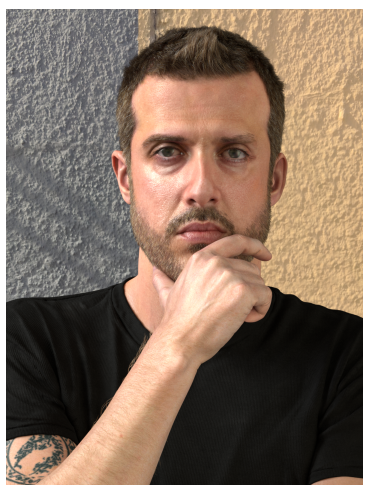

(c) digital character lit with $\mathbf{b}$

Figure 7: (a) In-the-wild input. (b) Estimated lighting. (c) A digital human rendered in V-Ray with the predicted illumination, composited into the scene. Model by Ian Spriggs.

\section{REFERENCES}

Dan A Calian, Jean-François Lalonde, Paulo Gotardo, Tomas Simon, Iain Matthews, and Kenny Mitchell. 2018. From Faces to Outdoor Light Probes. In Computer Graphics Forum, Vol. 37. Wiley Online Library, 51-61.

Paul Debevec. 1998. Rendering synthetic objects into real scenes: Bridging traditional and image-based graphics with global illumination and high dynamic range photography. In Proceedings of the 25th annual conference on Computer graphics and interactive techniques. ACM, 189-198.

Paul Debevec, Tim Hawkins, Chris Tchou, Haarm-Pieter Duiker, Westley Sarokin, and Mark Sagar. 2000. Acquiring the reflectance field of a human face. In Proceedings of the 27th annual conference on Computer graphics and interactive techniques. ACM Press/Addison-Wesley Publishing Co., 145-156.

Paul Debevec, Andreas Wenger, Chris Tchou, Andrew Gardner, Jamie Waese, and Tim Hawkins. 2002. A lighting reproduction approach to live-action compositing. ACM Transactions on Graphics (TOG) 21, 3 (2002), 547-556.

Bernhard Egger, Sandro Schönborn, Andreas Schneider, Adam Kortylewski, Andreas Morel-Forster, Clemens Blumer, and Thomas Vetter. 2018. Occlusion-aware 3d morphable models and an illumination prior for face image analysis. International fournal of Computer Vision 126, 12 (2018), 1269-1287.

Marc-André Gardner, Kalyan Sunkavalli, Ersin Yumer, Xiaohui Shen, Emiliano Gambaretto, Christian Gagné, and Jean-François Lalonde. 2017. Learning to Predict Indoor Illumination from a Single Image. ACM Trans. Graph. 36, 6, Article 176 (Nov.

2017), 14 pages. https://doi.org/10.1145/3130800.3130891

Yannick Hold-Geoffroy, Kalyan Sunkavalli, Sunil Hadap, Emiliano Gambaretto, and Jean-François Lalonde. 2017. Deep outdoor illumination estimation. In IEEE International Conference on Computer Vision and Pattern Recognition, Vol. 2.

Animesh Karnewar and Oliver Wang. 2020. Msg-gan: Multi-scale gradients for generative adversarial networks. In Proceedings of the IEEE/CVF Conference on Computer Vision and Pattern Recognition. 7799-7808.

Sebastian B Knorr and Daniel Kurz. 2014. Real-time illumination estimation from faces for coherent rendering. In 2014 IEEE International Symposium on Mixed and Augmented Reality (ISMAR). IEEE, 113-122.

Chloe LeGendre, Wan-Chun Ma, Graham Fyffe, John Flynn, Laurent Charbonnel, Jay Busch, and Paul Debevec. 2019. Deeplight: Learning illumination for unconstrained mobile mixed reality. In Proceedings of the IEEE Conference on Computer Vision and Pattern Recognition. 5918-5928.

Stephen R Marschner and Donald P Greenberg. 1997. Inverse lighting for photography. In Color and Imaging Conference, Vol. 1997. Society for Imaging Science and Technology, 262-265.

Ravi Ramamoorthi and Pat Hanrahan. 2001. On the relationship between radiance and irradiance: determining the illumination from images of a convex Lambertian object. FOSA A 18, 10 (2001), 2448-2459.

Soumyadip Sengupta, Angjoo Kanazawa, Carlos D Castillo, and David W Jacobs. 2018. SfSNet: learning shape, reflectance and illuminance of faces in the wild. In Proceedings of the IEEE Conference on Computer Vision and Pattern Recognition (CVPR). 6296-6305.

Hyunjung Shim. 2012. Faces as light probes for relighting. Optical Engineering 51, 7 (2012), 077002.

Tiancheng Sun, Jonathan T. Barron, Yun-Ta Tsai, Zexiang Xu, Xueming Yu, Graham Fyffe, Christoph Rhemann, Jay Busch, Paul Debevec, and Ravi Ramamoorthi. 2019. Single Image Portrait Relighting. ACM Trans. Graph. 38, 4, Article 79 (July 2019), 12 pages. https://doi.org/10.1145/3306346.3323008

Ayush Tewari, Michael Zollhöfer, Hyeongwoo Kim, Pablo Garrido, Florian Bernard, Patrick Pérez, and Christian Theobalt. 2017. Mofa: Model-based deep convolutional face autoencoder for unsupervised monocular reconstruction. In The IEEE International Conference on Computer Vision (ICCV), Vol. 2. 5.

Renjiao Yi, Chenyang Zhu, Ping Tan, and Stephen Lin. 2018. Faces as lighting probes via unsupervised deep highlight extraction. In Proceedings of the European Conference on Computer Vision (ECCV). 317-333.

Hao Zhou, Jin Sun, Yaser Yacoob, and David W Jacobs. 2018. Label Denoising Adversarial Network (LDAN) for Inverse Lighting of Faces. In Proceedings of the IEEE Conference on Computer Vision and Pattern Recognition. 6238-6247. 